\title{
Inflation and income inequality in a variety-expansion growth model with menu costs
}

\author{
Zhijie Zheng* \\ Beijing Normal University at Zhuhai University of Southampton \\ Yibai Yang \\ University of Macau
}

\begin{abstract}
This study explores the effect of inflation on income inequality in a growth model with variety expansion and menu costs. Inflation is found to affect income inequality through the asset-value effect in addition to the interest-rate effect. The numerical analysis shows that higher inflation makes these effects negative, so it stifles economic growth and mitigates income inequality.
\end{abstract}

JEL classification: $\mathrm{O}_{30} ; \mathrm{O}_{40} ; \mathrm{E}_{5} 8 ; \mathrm{D}_{30}$

Keywords: Inflation; Income inequality; Menu costs; Variety expansion

*Center for Innovation and Development Studies, Beijing Normal University at Zhuhai, 519087, China. Email address: zhengzhijie1919@gmail.com. 


\section{Introduction}

The rising degree of income inequality has become an important economic phenomenon in many developed countries over the past few decades (Saez and Zucman, 2016). Therefore, for explanation, recent studies have been attempting to investigate the relationship between income inequality and a variety of policy regimes. ${ }^{1}$ Among the existing policy regimes, monetary policy has been receiving increasing attention. Given that monetary policy has been used for price stability and economic growth, it can generate different impacts on different income groups in an economy, which may intensify or mitigate the degree of income inequality. ${ }^{2}$

This study adds to the current debate by revisiting the effect of inflation on economic growth and income inequality in an innovative-driven growth framework as in Chu, Cozzi, Fan, Furukawa and Liao (2019) but incorporates menu costs as in Oikawa and Ueda (2018). The novel contribution of this study is to analyze the monetary effect on income inequality in a varietyexpansion model developed by Romer (1990) and Rivera-Batiz and Romer (1991), with a labequipment R\&D specification. As Piketty, Saez and Stantcheva (2014) argues, an unequal distribution of wealth is an important source of income inequality, so we consider heterogeneous households with different asset holdings. In this model, since wage income is equally distributed across households, inflation affects income inequality by altering households' asset income. The overall impact of inflation operates through (a) changing the rate of economic growth and then the rate of real interest (i.e., the interest-rate channel) and (b) changing the value of wage and then the ratio of asset income relative to wage income (i.e., the asset-value channel). The numerical results show that in the case of higher inflation, the interest-rate effect is negative, whereas the asset-value effect can be either negative or positive. Therefore, this could lead to a negative or potentially positive impact of inflation on income inequality, depending on certain parameter values. ${ }^{3}$ This mechanism for the inflation-inequality relation differs from that in Zheng (2020), who explores a similar question in a Schumpeterian growth model with a knowledge-based specification. ${ }^{4}$ We find that such a difference arises from the distinction between the knowledge-based specification and the lab-equipment specification (instead of the dissimilarity between qualityimproving innovations and variety-expanding innovations), which determines the asset-value channel to be active or not. The above findings are robust to the extension with elastic labor supply, as shown in Appendix B.

\footnotetext{
${ }^{1}$ See, for example, Piketty, Saez and Stantcheva (2014) for tax policy and Chu and Cozzi (2018) for patent policy.

${ }^{2}$ See Coibion, Gorodnichenko, Kueng and Silvia (2017) for detailed discussions on the connection between monetary policy and income inequality.

3 See Appendix A for more details.

${ }^{4}$ In Appendix C, we also extend the benchmark model to a knowledge-based specification.
} 


\section{Model}

Suppose that there is a cashless economy in which the central bank directly controls the inflation rate. Throughout the analysis, we focus on the balanced growth path (BGP). Denote by $g$ the growth rate of a real variable, and by $\pi$ the growth rate of an aggregate price index.

\subsection{Households}

There is a unit continuum of infinitely-lived households indexed by $h \in[0,1]$. Households have the same preferences over consumption $c_{t^{\prime}}$ but possess different levels of asset holding. The lifetime utility of household $h$ is given by

$$
U_{t}=\int_{t}^{\infty} e^{-\rho\left(t^{\prime}-t\right)} \ln c_{t^{\prime}}(h) d t^{\prime},
$$

where $\rho>0$ is the subjective discount rate. Household $h$ 's asset-accumulation equation is

$$
\dot{a}_{t}(h)=r_{t} a_{t}(h)+w_{t}-c_{t}(h),
$$

where $a_{t}(h)$ is the real asset value and $r_{t}$ is the real interest rate. Households inelastically supply one unit of labor and earn the real wage at a rate of $w_{t}$. Solving the standard utility-maximization problem yields the intertemporal optimal condition for household $h$ :

$$
\dot{c}_{t}(h) / c_{t}(h)=r_{t}-\rho .
$$

Thus, the total consumption by all households, defined as $c_{t} \equiv \int_{0}^{1} c_{t}(h) d h$, also grows over time at the same rate of real consumption such that $\dot{c}_{t} / c_{t}=\dot{c}_{t}(h) / c_{t}(h)=r_{t}-\rho$.

\subsection{Final goods}

Final goods, $y_{t}$, are produced competitively by using labor and a continuum of intermediate goods according to

$$
y_{t}=l_{t}^{\alpha} \int_{0}^{N_{t}}\left[x_{t}(j)\right]^{1-\alpha} d j,
$$

where $N_{t}$ is the measure of varieties of intermediate at time $t . l_{t}$ is the labor devoted to producing final goods. Since labor is only used in production, $l_{t}$ will equal to unity. $x_{t}(j)$ is the quantity demanded of intermediate goods $j \in[0,1]$ and $\alpha \in(0,1)$ is a parameter that determines the elasticity of substitution between the varieties of intermediate goods. Solving the 
profit-maximization problem yields the demand function for $x_{t}(j)$ :

$$
x_{t}(j)=(1-\alpha)^{\frac{1}{\alpha}}\left(\frac{P_{t}}{p_{t}(j)}\right)^{\frac{1}{\alpha}}
$$

where $p_{t}(j)$ is the price of $x_{t}(j)$ and $P_{t}$ is the price of final goods in addition to the aggregate price index. Moreover, the inverse demand function for $l_{t}$ is

$$
w_{t}=\alpha \int_{0}^{N_{t}}\left[x_{t}(j)\right]^{1-\alpha} d j
$$

\subsection{Intermediate goods}

Intermediate goods in industry $j$ are manufactured by a monopolist who uses one unit of final good as the production input. The real-period profit of the monopolist is

$$
\Pi_{t}\left(p_{t}(j)\right)=\frac{\left[p_{t}(j)-P_{t}\right] x_{t}(j)}{P_{t}}=\frac{\xi_{t}(j)-P}{\left[\xi_{t}(j)\right]^{1 / \alpha}}(1-\alpha)^{1 / \alpha} P^{(1 / \alpha-1)}=\frac{\xi_{t}(j)-1}{\left[\xi_{t}(j)\right]^{1 / \alpha}}(1-\alpha)^{1 / \alpha},
$$

where $\xi_{t}(j)=p_{t}(j) e^{-\pi t}$ is the real price of $x_{t}(j) . P$ is the price of final goods at time 0 and is normalized to one. We follow Goh and Olivier (2002) to assume that the patent breadth is incomplete so that each monopolist charges a limited price to exclude the competition of potential imitators. Accordingly, the profit-maximizing price is determined by the markup $(1<$ $\mu<1 /(1-\alpha))$ such that $\xi^{*}=\mu .^{5}$

We assume that when firms change their prices in period $t$, they need to incur $\kappa>0$ units of final goods as menu costs, which makes sticky the price of each intermediate good. Moreover, firms also pay menu costs upon entry. The expression $\xi_{t}(j)=p_{t}(j) e^{-\pi t}$ indicates that for any positive inflation rate $\pi>0$, the real price of intermediate goods decreases at the rate of $\pi$ for the increase in the price of final goods. ${ }^{6}$ Suppose that in period $t_{i+1}$ for $i=0,1,2, \cdots$, firms pay menu costs and reset the real price at $\xi_{i+1}$. Then the real present value of a monopolist upon entry is given by

$$
V_{t}(j)=\sum_{i=0}^{\infty}\left\{\int_{t_{i}}^{t_{i+1}} \Pi_{t^{\prime}}\left(\xi_{i} e^{-\pi\left(t^{\prime}-t_{i}\right)}\right) e^{-r\left(t^{\prime}-t\right)} d t^{\prime}-\kappa e^{-r\left(t_{i+1}-t\right)}\right\}-\kappa,
$$

where $\xi_{t}=\xi_{i} e^{-\pi\left(t-t_{i}\right)}$, and $t_{i}$ is the timing of price changes with $t_{0}=t$. As shown in Sheshinski and Weiss (1977) and Oikawa and Ueda (2018), the optimal pricing strategy for firms is to obey

\footnotetext{
5 The consideration of incomplete patent protection simplifies the pricing strategy of monopolists.

${ }^{6}$ We restrict our analysis to the case of $\pi>0$. When $\pi<0$, the real price of intermediate goods $\xi_{t}(j)$ rises over time. This case becomes less attractive, however, given our assumption of incomplete patent breadth, under which $\xi_{t}(j)$ should be lower than $\mu$. When $\pi=0, \xi_{t}$ is constant over time and firms have no incentive to change prices.
} 
the following $S$ s rule, with the maximum and minimum price, $S$ and $s$, respectively, given by

$$
S=\xi^{*}=\mu ; \quad \text { and } \quad s=S e^{-\pi \Delta},
$$

where $\Delta$ denotes the time interval between $s$ and $S$. Monopolists that start manufacturing in period $t$ set the real price at $S$ initially, and their firm value is now described by

$$
\begin{aligned}
V_{t}(j) & =\sum_{i=0}^{\infty}\left\{\int_{t+i \Delta}^{t+(i+1) \Delta} \Pi_{t^{\prime}}\left(S e^{-\pi\left(t^{\prime}-t-i \Delta\right)}\right) e^{-r\left(t^{\prime}-t\right)} d t^{\prime}-\kappa e^{-r(t+(i+1) \Delta-t)}\right\}-\kappa \\
& =\sum_{i=0}^{\infty}\left\{\int_{0}^{\Delta} \Pi_{t^{\prime \prime}+i \Delta+t}\left(S e^{-\pi t^{\prime \prime}}\right) e^{-r\left(t^{\prime \prime}+i \Delta\right)} d t^{\prime \prime}-\kappa e^{-r(i+1) \Delta}\right\}-\kappa \\
& =\sum_{i=0}^{\infty} e^{-i r \Delta}\left\{\int_{0}^{\Delta} \Pi\left(S e^{-\pi t^{\prime \prime}}\right) e^{-r t^{\prime \prime}} d t^{\prime \prime}-\kappa e^{-r \Delta}\right\}-\kappa \\
& =\frac{1}{1-e^{-r \Delta}}\left\{\int_{0}^{\Delta}\left[\left(S e^{-\pi t^{\prime \prime}}\right)^{1-1 / \alpha}-\left(S e^{-\pi t^{\prime \prime}}\right)^{-1 / \alpha}\right] e^{-r t^{\prime \prime}}(1-\alpha)^{1 / \alpha} d t^{\prime \prime}-\kappa e^{-r \Delta}\right\}-\kappa \\
& =\frac{[(1-\alpha) / \mu]^{1 / \alpha}}{\left(1-e^{-r \Delta}\right)}\left\{\frac{\mu\left[1-e^{-\left(r-\frac{1-\alpha}{\alpha} \pi\right) \Delta}\right]}{r-\frac{1-\alpha}{\alpha} \pi}-\frac{1-e^{-\left(r-\frac{\pi}{\alpha}\right) \Delta}}{r-\frac{\pi}{\alpha}}\right\}-\frac{\kappa}{1-e^{-r \Delta}} \equiv v_{t},
\end{aligned}
$$

where (7) and $S=\mu$ in (9) have been used. Notice that the firm value in (10) (i.e., $v_{t}$ ) is identical across industries $j \in[0,1]$. Hence, the optimal time interval $\Delta=\Delta(g, \pi)$ maximizing $v_{t}$ is determined by

$$
\frac{d v_{t}}{d \Delta}=0 \Leftrightarrow r v_{t}=\left(\frac{1-\alpha}{\mu}\right)^{\frac{1}{\alpha}} e^{\frac{\pi}{\alpha} \Delta}\left(\mu e^{-\pi \Delta}-1\right) .
$$

\section{$2.4 R \& D$}

New innovations for each variety are invented by a unit continuum of R\&D firms indexed by $\varepsilon \in[0,1]$. Each firm purchases $R_{t}(\varepsilon)$ units of final output for R\&D. The real expected profit of firm $\varepsilon$ is $v_{t} \dot{N}_{t}(\varepsilon)-R_{t}(\varepsilon)$, where $\dot{N}_{t}(\varepsilon)=\beta R_{t}(\varepsilon)$ is the number of inventions created by firm $\varepsilon$ and $\beta>0$ is a productivity parameter. Given the unit continuum of R\&D firms, we have $\dot{N}_{t}=\dot{N}_{t}(\varepsilon)$ in equilibrium. Therefore, the aggregate growth rate of innovation is $g=\dot{N}_{t} / N_{t}=\beta R_{t} / N_{t}$. Assume that the research sector is perfectly competitive with free entry. Then the flow of an R\&D firm's real profit must be zero, implying

$$
v_{t}=1 / \beta
$$




\section{Stationary Equilibrium}

Denote the total menu costs, aggregate level of asset holdings, firm values, intermediate goods and R\&D expenditure by $z_{t}, a_{t}=\int_{0}^{1} a_{t}(h) d h, V_{t}=\int_{0}^{N_{t}} V_{t}(j) d j=N_{t} v_{t}, x_{t}=\int_{0}^{N_{t}} x_{t}(j) d j$ and $R_{t}=\int_{0}^{1} R_{t}(\varepsilon) d \varepsilon$, respectively. The general equilibrium is defined as follows.

Definition 1. The general equilibrium consists of sequences of aggregate variables $\left\{a_{t}, l_{t}, c_{t}, y_{t}, x_{t}, z_{t}, R_{t}\right\}_{t=0}^{\infty}$, intermediate-goods firm $j^{\prime}$ s decisions $\left\{\xi_{t}(j), \Delta\right\}_{t=0}^{\infty}$, RED firm $\varepsilon^{\prime}$ s decisions $\left\{R_{t}(\varepsilon)\right\}_{t=0}^{\infty}$, household $h^{\prime} s$ choices $\left\{a_{t}(h), c_{t}(h)\right\}_{t=0}^{\infty}$, and aggregate prices $\left\{P_{t}, p_{t}(j), \xi_{t}(j), w_{t}, r_{t}, V_{t}\right\}_{t=0}^{\infty}$, for $h \in[0,1], j \in[0,1]$ and $\varepsilon \in[0,1]$ such that households and firms solve their optimization problems, and all markets clear. That is, the asset market clears such that $V_{t}=a_{t}$. The labor market clears such that $l_{t}=1$. The final-goods market clears such that

$$
c_{t}+x_{t}+z_{t}+R_{t}=y_{t}
$$

The resource constraint in (13) shows that the aggregate demand of final goods (for consumption, producing intermediate goods, paying menu costs, and RED spending) equals the aggregate production of final goods.

To solve the equilibrium, we follow Arawatari, Hori and Mino (2018) to derive the equilibrium expressions of various variables as follow.

Lemma 1. The equilibrium wage rate, aggregate intermediate goods, total menu costs, and consumption are given by

$$
\begin{gathered}
w_{t}=\alpha(1-\alpha)^{\frac{1-\alpha}{\alpha}} \frac{g N_{t}}{1-e^{-g \Delta}} \underbrace{\frac{\left[e^{\left(\frac{1-\alpha}{\alpha} \pi-g\right) \Delta}-1\right] \mu^{-\frac{1-\alpha}{\alpha}}}{(1-\alpha) \pi / \alpha-g}}_{\Omega(\Delta)} . \\
z_{t}=\frac{\kappa g N_{t}}{1-e^{-g \Delta} .} \\
x_{t}=(1-\alpha)^{\frac{1}{\alpha}} \frac{g N_{t}}{1-e^{-g \Delta} \underbrace{\frac{\left[e^{(\pi / \alpha-g) \Delta}-1\right] \mu^{-\frac{1}{\alpha}}}{\pi / \alpha-g}}_{\Phi(\Delta)} .} \underbrace{}_{c_{t}=\frac{g N_{t}}{1-e^{-g \Delta}}\left[(1-\alpha)^{\frac{1-\alpha}{\alpha}} \Omega(\Delta)-(1-\alpha)^{\frac{1}{\alpha}} \Phi(\Delta)-\left(1-e^{-g \Delta}\right) / \beta-\kappa\right]}
\end{gathered}
$$

Proof. See the Appendix A.1.

Equations (14) - (17) show that the equilibrium allocations are functions of endogenous variables $\Delta$ and $g$, for any given $\pi$. Combining (I1) with (12) and (10) with (12), respectively, this model can be reduced to a system of two equations involving $\Delta$ and $g$ :

$$
\frac{\rho+g}{\beta}=\left(\frac{1-\alpha}{\mu}\right)^{\frac{1}{\alpha}} e^{\frac{\pi}{\alpha} \Delta}\left(\mu e^{-\pi \Delta}-1\right),
$$




$$
1 / \beta=\frac{[(1-\alpha) / \mu]^{1 / \alpha}}{\left[1-e^{-(\rho+g) \Delta}\right]}\left\{\frac{\mu\left[1-e^{-\left(\rho+g-\frac{1-\alpha}{\alpha} \pi\right) \Delta}\right]}{\rho+g-\frac{1-\alpha}{\alpha} \pi}-\frac{1-e^{-\left(\rho+g-\frac{\pi}{\alpha}\right) \Delta}}{\rho+g-\frac{\pi}{\alpha}}\right\}-\frac{\kappa}{1-e^{-(\rho+g) \Delta}} .
$$

(18) and (19) determines the equilibrium values of $\Delta$ and $g$, which in turns determines the equilibrium allocations as specified by (14) - (17).

\section{Inflation and income inequality}

In this section, we first show that the asset distribution on the BGP is stationary in this model, and then examine the effect of inflation on income inequality.

\subsection{Asset distribution}

Define $\theta_{a, 0}(h) \equiv a_{0}(h) / a_{0}$ as the initial share of asset owned by household $h$ at time 0 , which is exogenously given and follows a general distribution function with a mean of one and a standard deviation of $\sigma_{a}>0$. Aggregating (2) by $h$ yields the aggregate asset-accumulation function given by $\dot{a}_{t}=r_{t} a_{t}+w_{t}-c_{t}$. Thus the dynamics of household $h^{\prime}$ s asset share at time $t$ is

$$
\frac{\dot{\theta}_{a, t}(h)}{\theta_{a, t}(h)}=\frac{\dot{a}_{t}(h)}{a_{t}(h)}-\frac{\dot{a}_{t}}{a_{t}}=\frac{c_{t}-w_{t}}{a_{t}}-\frac{c_{t}(h)-w_{t}}{a_{t}(h)} \Leftrightarrow \dot{\theta}_{a, t}(h)=\frac{c_{t}-w_{t}}{a_{t}} \theta_{a, t}(h)-\frac{c_{t} \theta_{c, t}(h)-w_{t}}{a_{t}},
$$

where $\theta_{c, t}(h) \equiv c_{t}(h) / c_{t}$ is the share of consumption by household $h$. Using $\dot{c}_{t} / c_{t}=\dot{c}_{t}(h) / c_{t}(h)$ from (3), we show that $\theta_{c, t}(h)$ is time invariant and equals to $\theta_{c}(h)$ for all $t$ because $\dot{\theta}_{c, t}(h) / \theta_{c, t}(h)=$ $\dot{c}_{t}(h) / c_{t}(h)-\dot{c}_{t} / c_{t}=0$. Given that the real economic variables $\left\{c_{t}, w_{t}, a_{t}\right\}$ all grow at the rate of $g$ along the BGP, $\dot{a}_{t}=r_{t} a_{t}+w_{t}-c_{t}$ is reduced to $c_{t}-w_{t}=\rho a_{t}$ and thus the coefficient associated with $\theta_{a, t}(h)$ in (20) is reduced to $\rho>0$. Therefore, the one-dimensional differential equation as described in (20) indicates that $\dot{\theta}_{a, t}(h)$ must be zero for all $t$ to be consistent with the long-run stability given that $\theta_{a, t}(h)$ is a state variable. ${ }^{7}$ Moreover, applying $\dot{\theta}_{a, t}(h)=0$ into (20) yields

$$
\theta_{c, t}(h)-1=\frac{\rho a_{t}}{c_{t}}\left[\theta_{a, 0}(h)-1\right]
$$

\subsection{Income distribution}

The real income earned by household $h$ and the aggregate real income earned by all households are $I_{t}(h)=r_{t} a_{t}(h)+w_{t}$ and $I_{t}=r_{t} a_{t}+w_{t}$, respectively. Combining $I_{t}(h)$ and $I_{t}$ yields the share

\footnotetext{
7We need to maintain caution here because the model may feature transitional dynamics. The asset distribution may deviate from the one on the old BGP, following a change of the inflation rate. Due to the complexity of characterizing transitional dynamics, however, we assume here that inflation has a negligible effect on asset inequality and take - as given - the degree of asset inequality when analyzing the effects of inflation on income inequality.
} 
of income by household $h$ :

$$
\theta_{I, t}(h) \equiv \frac{I_{t}(h)}{I_{t}}=\frac{r_{t} a_{t}(h)+w_{t}}{r_{t} a_{t}+w_{t}}=\frac{r_{t} a_{t} \theta_{a, 0}(h)+w_{t}}{r_{t} a_{t}+w_{t}}
$$

where $\theta_{a, t}(h)=\theta_{a, 0}(h)$ has been used. The distribution function of income share $\theta_{I, t}(h)$ has a mean of one and the following standard deviation:

$$
\sigma_{I, t}=\sigma_{I} \equiv \sqrt{\int_{0}^{1}\left[\theta_{I, t}(h)-1\right]^{2} d h}=\frac{r_{t} a_{t} / w_{t}}{1+r_{t} a_{t} / w_{t}} \sigma_{a},
$$

which is an increasing function of the asset income relative to wage income $\left(r_{t} a_{t} / w_{t}\right)$ as in Chu, Cozzi, Fan, Furukawa and Liao (2019) and Zheng (2020). It is because an unequal distribution of wealth is the source of income inequality. The aggregate effect of inflation on income inequality can be decomposed into two channels: the interest-rate effect (i.e., $r_{t}$ ) and the asset-value effect (i.e., $\left.a_{t} / w_{t}\right)$, as identified by Chu and Cozzi (2018). First, altering the inflation rate $\pi$ has an impact on the steady-state interest rate $r$ since the growth rate $g$ determined by (18) and (19) is changed. Furthermore, the impact of $\pi$ on the asset value relative to the wage rate in the steady state can be obtained by using the asset-market clearing condition and free-entry condition in the R\&D sector:

$$
\frac{a_{t}}{w_{t}}=\frac{N_{t} v_{t}}{w_{t}}=\left(\frac{\mu}{1-\alpha}\right)^{\frac{1-\alpha}{\alpha}}\left[\frac{\left(\frac{1-\alpha}{\alpha}\right) \pi-g}{\alpha \beta g}\right] \frac{e^{g \Delta}-1}{e^{\frac{1-\alpha}{\alpha} \pi \Delta}-e^{g \Delta}} .
$$

Equation (24) shows that $a_{t} / w_{t}$ relies on the impact of $\pi$ directly in addition to the impact of $\pi$ indirectly through affecting $\Delta$ and $g$ determined by (18) and (19).

Due to the complexity and analytical ambiguity, we resort to a numerical exercise to evaluate the effect of inflation on income inequality. Our benchmark parameter specification is $\rho=0.02$, $\alpha=0.6$ and $\mu=1.2$, which are conventional values in the literature. We follow Midrigan (2011) to set the menu cost parameter to $\kappa=0.022$. The productivity parameter $\beta$ is calibrated to 1.3470 by matching the standard moments of the inflation rate $\pi=2.5 \%$ and the growth rate $g=2 \%$.

Given the above parametrization, Figure $1 b$ shows that the economic growth rate is monotonically decreasing in the inflation rate. ${ }^{8}$ Intuitively, when the nominal rigidity is present in the economy, a higher inflation rate leads the real prices of monopolistic firms to decrease faster, as shown in Figure 1a. This causes firms to adjust their prices more frequently and pay more menu costs. Therefore, higher inflation tends to reduce the firm value. Nevertheless, given that free entry to the R\&D sector implies a constant firm value over time as shown in (12), a lower interest rate responds to higher inflation to maintain the constant firm value. As a result, higher inflation stifles economic growth according to (3).

\footnotetext{
${ }^{8}$ Our numerical analysis is restricted for a positive growth rate, implying a maximum inflation rate of $21.17 \%$.
} 

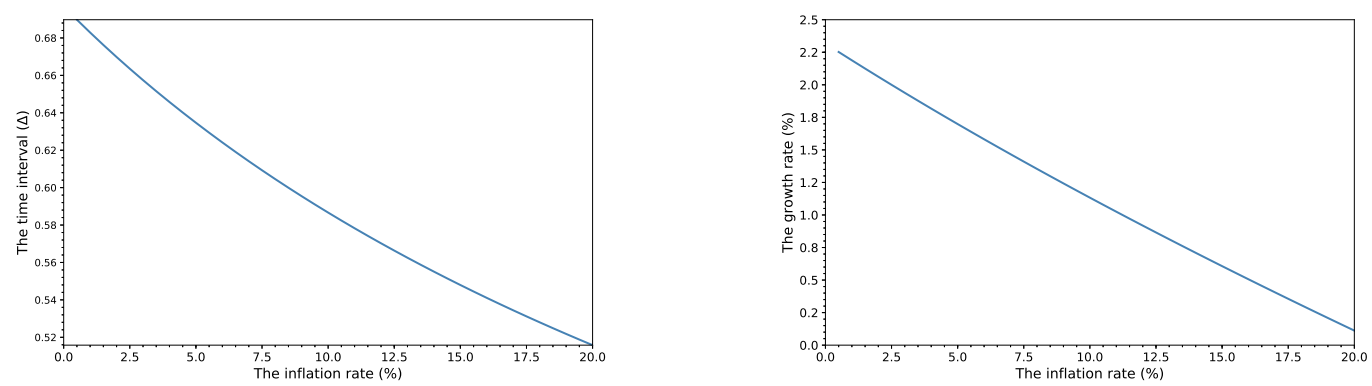

Figure 1: (a) Inflation and time interval; (b) Inflation and economic growth.

In addition to the negative interest-rate effect of inflation by reducing the economic growth rate, a higher inflation rate has a negative effect on the asset value (relative to the wage rate), as shown in Figure 2a. This is because a higher $\pi$ tends to raise the steady-state level of the productivity-adjusted wage rate $w_{t} / N_{t}$ in (24). ${ }^{9}$ Therefore, both the interest-rate effect and the asset-value effect contribute to a monotonically decreasing effect of inflation on the degree of income inequality, as illustrated in Figure 2b. Nevertheless, as shown in Appendix A.2, under a larger $\kappa$ and much lower $\mu$, the asset-value effect and the effect of inflation on income inequality can be positive.
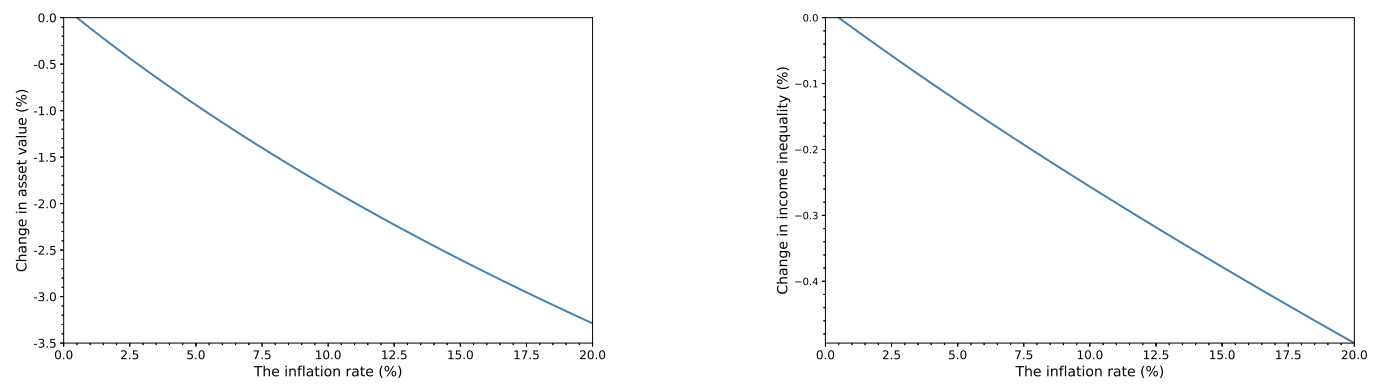

Figure 2: (a) Inflation and asset value; (b) Inflation and income inequality.

${ }^{9}$ Higher inflation $\pi$ decreases the real prices $\xi_{t}(j)$ of monopolistic firms. However, the free-entry condition in (12) requires a constant firm value $v_{t}$ of these producers. Given the interest rate $r_{t}$ and the technology level $N_{t}$, the monopolistic firms have to supply more intermediate goods $x_{t}(j)$ to maintain their profit levels, which tends to raise the wage rate $w_{t}$ as shown in (6). 


\section{Conclusion}

This study explores the effect of inflation on income inequality in a monetary version of the lab-equipment model of growth with variety expansion and menu costs. Similar to Chu, Cozzi, Fan, Furukawa and Liao (2019), who incorporate money demand through CIA constraints on consumption and R\&D, the current study reveals that inflation can also affect income inequality through both the interest-rate effect and the asset-value effect in a cashless economy. ${ }^{10}$ In the numerical analysis, under higher inflation, the interest-rate effect is negative, but the asset-value effect and the impact of inflation on income inequality could be negative or positive, subject to values of parameters. Therefore, comparing the current study to Zheng (2020) implies that the process of innovation (i.e., variety expansion versus quality improvement) and the R\&D specification (i.e., lab-equipment versus knowledge-driven) play a crucial role in determining how inflation affects income inequality in an R\&D-based growth framework.

\section{Acknowledgment}

We sincerely thank an anonymous referee, Prof. Eric Young (the Associate Editor) and Prof. Pierre-Daniel Sarte (the Editor) for many helpful comments. Their intuitions have certainly improved the quality of inferences and claim we make in the paper. We take sole responsibility for any error and omissions.

\section{Appendix A}

\section{A.1 Proof of Lemma 1}

We follow the appendix in Arawatari, Hori and Mino (2018) to prove Lemma 1. Recall that the wage rate is

$$
w_{t}=\alpha \int_{0}^{N_{t}}\left(\frac{1}{x_{t}(j)}\right)^{\alpha-1} d j=\alpha(1-\alpha)^{\frac{1-\alpha}{\alpha}} \int_{0}^{N_{t}}\left[\xi_{t}(j)\right]^{-\frac{1-\alpha}{\alpha}} d j
$$

where (5) and $\xi_{t}(j)=p_{t}(j) / P_{t}$ have been used. Consider firms whose age is $\gamma=a+s \Delta$, where $a \in[0, \Delta)$. The number of such firms is given by

$$
\dot{N}_{t-\gamma}=g N_{t-\gamma}=g N_{t-(a+s \Delta)}=g N_{t-s \Delta} e^{-g a} .
$$

\footnotetext{
${ }^{10}$ Nevertheless, Appendix C shows that in the knowledge-based version of the benchmark model, inflation affects income inequality only via the interest-rate effect but not through the asset-value effect, as in Zheng (2020).
} 
Remember that each monopolist follows the same pricing rule. Thus, we have

$$
\begin{aligned}
\int_{0}^{N_{t}}\left[\xi_{t}(j)\right]^{-\frac{1-\alpha}{\alpha}} d j & =\int_{0}^{\Delta}\left(\xi_{t-\gamma}\right)^{-\frac{1-\alpha}{\alpha}} g N_{t-\gamma} d \gamma \\
& +\int_{\Delta}^{2 \Delta}\left(\xi_{t-\gamma}\right)^{-\frac{1-\alpha}{\alpha}} g N_{t-\gamma} d \gamma+\cdots \\
& +\int_{s \Delta}^{(s+1) \Delta}\left(\xi_{t-\gamma}\right)^{-\frac{1-\alpha}{\alpha}} g N_{t-\gamma} d \gamma+\cdots \\
& =\sum_{s=0}^{\infty} \int_{s \Delta}^{(s+1) \Delta}\left(\xi_{t-(\gamma-s \Delta)}\right)^{-\frac{1-\alpha}{\alpha}} g N_{t-\gamma} d \gamma,
\end{aligned}
$$

where

$$
\begin{aligned}
\int_{s \Delta}^{(s+1) \Delta}\left(\xi_{t-(\gamma-s \Delta)}\right)^{-\frac{1-\alpha}{\alpha}} g N_{t-\gamma} d \gamma & =\int_{0}^{\Delta}\left(\xi_{t-a}\right)^{-\frac{1-\alpha}{\alpha}} g N_{t-(a+s \Delta)} d a \\
& =g N_{t-s \Delta} \int_{0}^{\Delta}\left(\xi_{t-a}\right)^{-\frac{1-\alpha}{\alpha}} e^{-g a} d a \\
& =g N_{t} e^{-g s \Delta} \int_{0}^{\Delta}\left(\xi_{t-a}\right)^{-\frac{1-\alpha}{\alpha}} e^{-g a} d a \\
& =g N_{t} e^{-g s \Delta} \int_{0}^{\Delta} \mu^{-\frac{1-\alpha}{\alpha}} e^{\frac{1-\alpha}{\alpha} \pi a} e^{-g a} d a \\
& =g N_{t} e^{-g s \Delta} \underbrace{\frac{\left[e^{\left(\frac{1-\alpha}{\alpha} \pi-g\right) \Delta}-1\right] \mu^{-\frac{1-\alpha}{\alpha}}}{(1-\alpha) \pi / \alpha-g}}_{\Omega(\Delta)}
\end{aligned}
$$

where we applied $\xi_{t-a}=\mu e^{-\pi a}$ in the forth equation of (A.4). Inserting (A.4) into (A.3) and plugging the resulting equation into (A.I) yield the steady state equilibrium wage rate in Lemma I such that

$$
w_{t}=\alpha(1-\alpha)^{\frac{1-\alpha}{\alpha}} \frac{g N_{t}}{1-e^{-g \Delta}} \Omega(\Delta) .
$$

Therefore, the aggregate output is expressed as

$$
y_{t}=\frac{w_{t} l_{t}}{\alpha}=\frac{w_{t}}{\alpha}=(1-\alpha)^{\frac{1-\alpha}{\alpha}} \frac{g N_{t}}{1-e^{-g \Delta}} \Omega(\Delta) .
$$


Similarly, the aggregate amount of intermediate goods is derived as

$$
\begin{aligned}
x_{t} & =\int_{0}^{N_{t}} x_{t}(j) d j=(1-\alpha)^{\frac{1}{\alpha}} \int_{0}^{N_{t}}\left[\xi_{t}(j)\right]^{-\frac{1}{\alpha}} d j \\
& =(1-\alpha)^{\frac{1}{\alpha}} \sum_{s=0}^{\infty} \int_{s \Delta}^{(s+1) \Delta}\left(\xi_{t-(\gamma-s \Delta)}\right)^{-\frac{1}{\alpha}} g N_{t-\gamma} d \gamma \\
& =(1-\alpha)^{\frac{1}{\alpha}} \sum_{s=0}^{\infty} g N_{t} e^{-g s \Delta} \int_{0}^{\Delta}\left(\xi_{t-a}\right)^{-\frac{1}{\alpha}} e^{-g a} d a \\
& =(1-\alpha)^{\frac{1}{\alpha}} \sum_{s=0}^{\infty} g N_{t} e^{-g s \Delta} \int_{0}^{\Delta} \mu^{-\frac{1}{\alpha}} e^{\frac{\pi}{\alpha} a} e^{-g a} d a \\
& =(1-\alpha)^{\frac{1}{\alpha}} \frac{g N_{t}}{1-e^{-g \Delta} \underbrace{\left[e^{(\pi / \alpha-g) \Delta}-1\right] \mu^{-\frac{1}{\alpha}}}_{\Phi(\Delta)}} .
\end{aligned}
$$

Moreover, at time $t$, new intermediate-goods firms, with an amount of $\dot{N}_{t}=g N_{t}$, incur menu costs of $\kappa$ to set their prices at entry. In addition, firms whose age is $s \Delta$ also pay menu costs to adjust their prices, where $s=0,1,2, \ldots$. Given that the number of these firms is $g N_{t-s \Delta}=$ $g N_{t} e^{-g s \Delta}$, the total menu costs are given by

$$
z_{t}=\sum_{s=0}^{\infty} g N_{t} e^{-g s \Delta} \mathcal{K}=\frac{\kappa g N_{t}}{1-e^{-g \Delta}}
$$

Substituting $R_{t}=g N_{t} / \beta$, (A.6), (A.7) and (A.8) into the final-goods market clearing condition (13) yields the aggregate level of consumption such that

$$
c_{t}=y_{t}-x_{t}-z_{t}-R_{t}=\frac{g N_{t}}{1-e^{-g \Delta}}\left[(1-\alpha)^{\frac{1-\alpha}{\alpha}} \Omega(\Delta)-(1-\alpha)^{\frac{1}{\alpha}} \Phi(\Delta)-\frac{1-e^{-g \Delta}}{\beta}-\kappa\right] .
$$

\section{A.2 Additional numerical results}

In this subsection, we show numerically that both the asset-value and inequality effect can be positive. When adjusting the markup value from the benchmark value to 1.1 and menu cost parameter $\kappa$ to 0.6 , there arises a positive effect of inflation on the asset value (relative to wage rate) as in Figure 3. Yet, the nexus between inflation and income inequality is still negative, which indicates that the negative interest-rate effect dominates the positive asset-value effect. In this case, the economic growth rate decreases very fast to zero. The intuition is straightforward. When the price-adjusting cost is sufficiently high, represented by $\kappa$, even for a small rise in the inflation rate, the cost for firms to change their prices is very high. As a result, firms' profits could decrease to zero, making R\&D unattractive and leading to no growth.

In this model, for a very large wide and reasonable range of parameter values, the relationship 


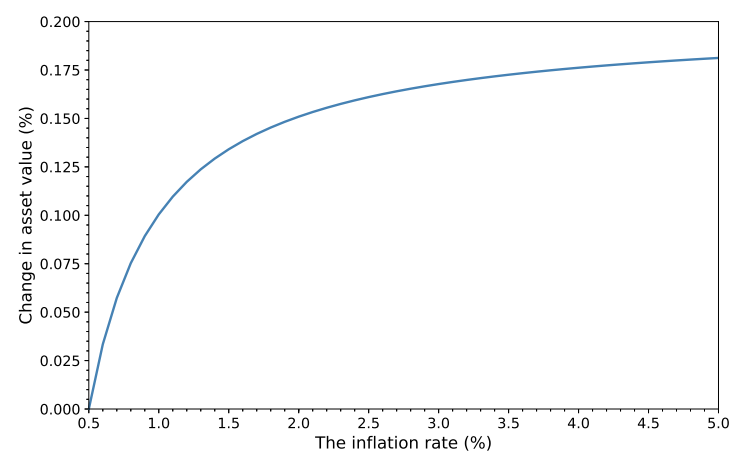

Figure 3: Inflation and asset value.

of inflation and inequality is negative. In order to show the mathematical possibility of a positive linkage, we fix $\kappa$ to 0.6 and set the markup to 0.2 , which is apparently implausible. Figure $4 \mathrm{a}$ and $4 \mathrm{~b}$ then show that the impact of inflation on the asset-value effect and income inequality become positive. Again, since this case is unrealistic, we intend to keep it only as a mathematical experiment.
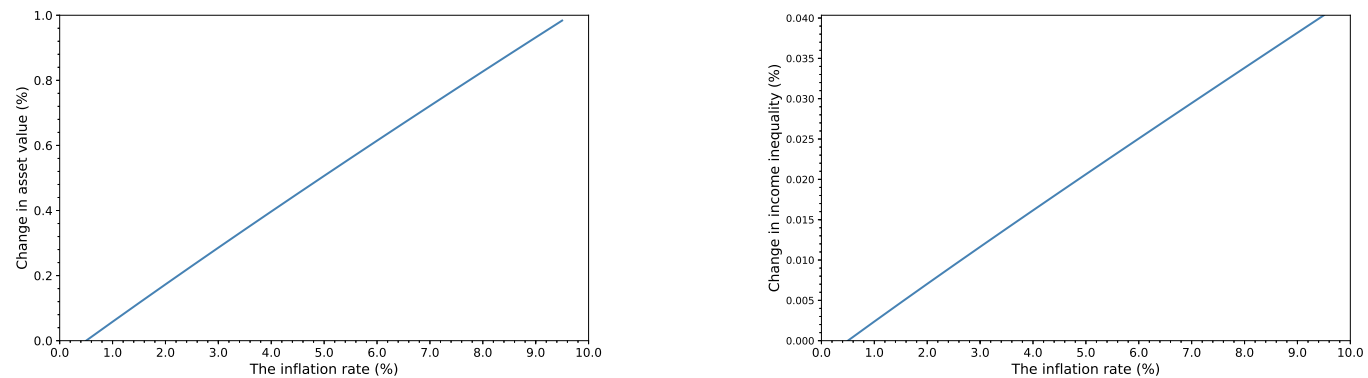

Figure 4: (a) Inflation and asset value; (b) Inflation and income inequality.

\section{Appendix B Extension of elastic labor supply}

In this appendix, we consider elastic labor supply by households. In this case, household $h$ 's utility function is given by

$$
U_{t}=\int_{t}^{\infty} e^{-\rho\left(t^{\prime}-t\right)}\left\{\ln c_{t^{\prime}}(h)+\varphi \ln \left[1-l_{t^{\prime}}(h)\right]\right\} d t^{\prime},
$$

where $\varphi>0$ measures household $h^{\prime}$ s preference of leisure. The asset-accumulation function is now given by $\dot{a}_{t}(h)=r_{t} a_{h}+w_{t} l_{t}(h)-c_{t}(h)$. Solving household $h^{\prime}$ s utility-maximizing problem 
yields

$$
w_{t}\left[1-l_{t}(h)\right]=\varphi c_{t}(h)
$$

and the Euler equation as in (3). The demand function of $x_{t}(j)$ in (5) and the wage rate in (6) are now given by, respectively,

$$
\begin{gathered}
x_{t}(j)=(1-\alpha)^{\frac{1}{\alpha}}\left(\frac{P_{t}}{p_{t}(j)}\right)^{\frac{1}{\alpha}} l_{t}, \\
w_{t}=\alpha \int_{0}^{N_{t}}\left(\frac{l_{t}}{x_{t}(j)}\right)^{\alpha-1} d j .
\end{gathered}
$$

The real-period profit function of incumbents is

$$
\Pi_{t}\left(p_{t}(j)\right)=\frac{\xi_{t}(j)-1}{\left[\xi_{t}(j)\right]^{1 / \alpha}}(1-\alpha)^{1 / \alpha} l_{t} .
$$

Since $l_{t}=l$ is constant on the steady state equilibrium, the firm value in (10) and the optimal time interval determined in (11) are, respectively, given by

$$
\begin{gathered}
V_{t}(j)=\frac{l[(1-\alpha) / \mu]^{1 / \alpha}}{\left(1-e^{-r \Delta}\right)}\left\{\frac{\mu\left[1-e^{-\left(r-\frac{1-\alpha}{\alpha} \pi\right) \Delta}\right]}{r-\frac{1-\alpha}{\alpha} \pi}-\frac{1-e^{-\left(r-\frac{\pi}{\alpha}\right) \Delta}}{r-\frac{\pi}{\alpha}}\right\}-\frac{\kappa}{1-e^{-r \Delta}}, \\
r V_{t}(j)=\left(\frac{1-\alpha}{\mu}\right)^{\frac{1}{\alpha}} e^{\frac{\pi}{\alpha} \Delta}\left(\mu e^{-\pi \Delta}-1\right) l .
\end{gathered}
$$

Moreover, following the derivations in Appendix A, we obtain

$$
\begin{gathered}
w_{t}=\alpha(1-\alpha)^{\frac{1-\alpha}{\alpha}} \frac{g N_{t}}{1-e^{-g \Delta}} \Omega(\Delta), \\
y_{t}=\frac{w_{t} l_{t}}{\alpha}=(1-\alpha)^{\frac{1-\alpha}{\alpha}} \frac{g N_{t}}{1-e^{-g \Delta}} \Omega(\Delta) l, \\
x_{t}=(1-\alpha)^{\frac{1}{\alpha}} \frac{g N_{t}}{1-e^{-g \Delta}} \frac{\left[e^{(\pi / \alpha-g) \Delta}-1\right] \mu^{-\frac{1}{\alpha}}}{\pi / \alpha-g},
\end{gathered}
$$

and the expressions of $z_{t}$ and $R_{t}$ remain unchanged. Substituting these equations into the aggregate consumption yields

$$
c_{t}=\frac{g N_{t}}{1-e^{-g \Delta}}\left\{(1-\alpha)^{\frac{1-\alpha}{\alpha}} \Omega(\Delta) l-(1-\alpha)^{\frac{1}{\alpha}} \Phi(\Delta) l-\frac{1-e^{-g \Delta}}{\beta}-\kappa\right\}
$$

The model is eventually reduced to a three-equations system of endogenous variables $\{l, \Delta, g\}$. 
The first equation is obtained by combining (B.2) with (B.II) such that

$$
\alpha \Omega(\Delta)-\varphi\left(\frac{1-e^{-g \Delta}}{\beta}+\kappa\right)(1-\alpha)^{\frac{1-\alpha}{\alpha}}=l[(\varphi+\alpha) \Omega(\Delta)-\varphi(1-\alpha) \Phi(\Delta)]
$$

Combining (12) with (B.7) yields the second equation such that

$$
\frac{\rho+g}{\beta}=\left(\frac{1-\alpha}{\mu}\right)^{\frac{1}{\alpha}} e^{\frac{\pi}{\alpha} \Delta}\left(\mu e^{-\pi \Delta}-1\right) l
$$

Combining (12) with (B.6) yields the third equation such that

$$
\frac{\left(1-e^{-r \Delta}\right)}{\beta}=l[(1-\alpha) / \mu]^{1 / \alpha}\left\{\frac{\mu\left[1-e^{-\left(r-\frac{1-\alpha}{\alpha} \pi\right) \Delta}\right]}{r-\frac{1-\alpha}{\alpha} \pi}-\frac{1-e^{-\left(r-\frac{\pi}{\alpha}\right) \Delta}}{r-\frac{\pi}{\alpha}}\right\}-\kappa .
$$

As for the effect of inflation on income inequality, income inequality is still an increasing function of $r_{t} a_{t} / w_{t}$ as in (23), which can be further decomposed into the interest-rate effect (i.e., $r$ ) and the asset-value effect (i.e., $a_{t} / w_{t}$ ). The expression of $a_{t} / w_{t}$ is same as (24). The expression of income deviation is now given by

$$
\sigma_{I}=\frac{(\rho+g+\varphi g) a_{t} / w_{t}}{(\rho+g+\varphi g) a_{t} / w_{t}+1} \sigma_{a}
$$

where $\varphi$ captures the effect of elastic labor supply.

Again, we numerically evaluate the effects of inflation on economic growth and income inequality in this extension. We find that the result is robust to the counterpart in the benchmark model. We use the standard moment of labor supply (i.e, $l=1 / 3$ ) to calibrate the newly added parameter $\varphi$. Other parameters (except $\beta$ ) remain unchanged as in the benchmark parametrization. Then, the calibrated values of parameters are given by $\varphi=1.5523$ and $\beta=14.3680$. Figure $5 \mathrm{a}$ and $5 \mathrm{~b}$ show that the rate of economic growth and the degree of income inequality are monotonically deceasing in the rate of inflation. ${ }^{11}$

\section{Appendix C Knowledge-based specification}

In this section, we consider a knowledge-based version of the benchmark model. The main modification is that entrepreneurs employ labors, instead of final goods, for performing R\&D. The discovery rate of new innovations is given by $\dot{N}_{t}=\beta N_{t} l_{r, t}$, where $l_{r, t}$ denotes the level of

\footnotetext{
${ }^{11}$ Given the calibrated parameters, the economic growth rate decreases to zero at the inflation rate of $4 \%$. Thus, the range of the inflation rate in this numerical analysis is restricted within $[0,0.04]$.
} 

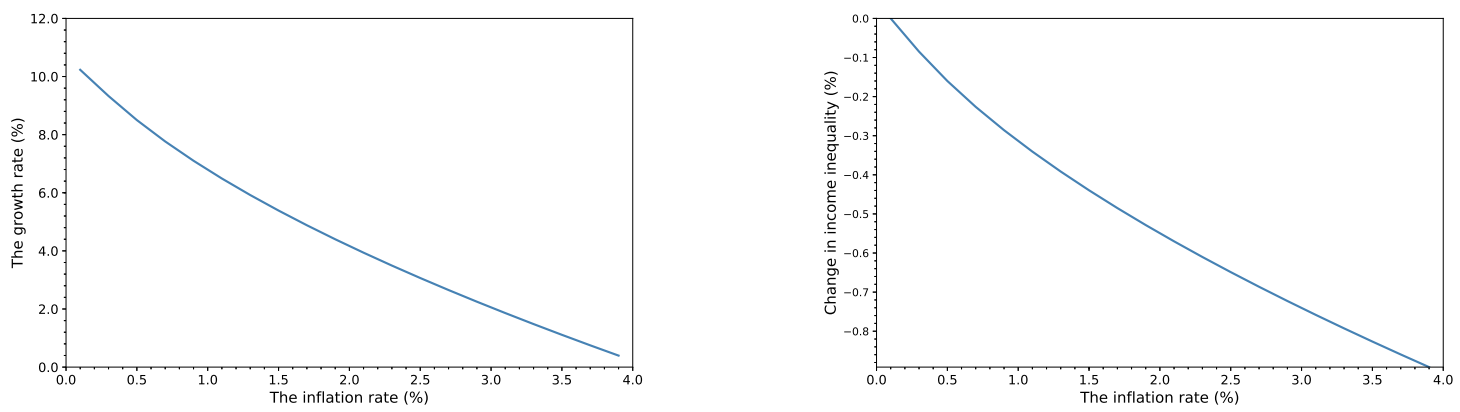

Figure 5: (a) Inflation and economic growth; (b) Inflation and income inequality.

labor employment hired for creating inventions. The free-entry condition to R\&D sector is

$$
\dot{N}_{t} V_{t}(j)=w_{t} l_{r t} \Leftrightarrow \beta V_{t}(j) N_{t}=w_{t} \Leftrightarrow V_{t}=w_{t} / \beta,
$$

where $V_{t}=\int_{0}^{N_{t}} V_{t}(j) d j$ is the aggregate firm value. For simplicity, we assume inelastic labor supply as in the benchmark framework. We denote by $l_{y, t}$ the level of labor used in final-goods production and thus obtain the labor-market clearing condition such that $l_{y, t}+l_{r, t}=1$. The real-period profit of the monopolistic intermediate-goods producers is given by

$$
\Pi_{t}\left(p_{t}(j)\right)=\frac{\xi_{t}(j)-1}{\left[\xi_{t}(j)\right]^{1 / \alpha}}(1-\alpha)^{1 / \alpha} l_{y, t}
$$

The firm value in (10) and the optimal time interval determined in (11) are now given by

$$
V_{t}(j)=\frac{l_{y t}[(1-\alpha) / \mu]^{1 / \alpha}}{\left(1-e^{-r \Delta}\right)}\left\{\frac{\mu\left[1-e^{-\left(r-\frac{1-\alpha}{\alpha} \pi\right) \Delta}\right]}{r-\frac{1-\alpha}{\alpha} \pi}-\frac{1-e^{-\left(r-\frac{\pi}{\alpha}\right) \Delta}}{r-\frac{\pi}{\alpha}}\right\}-\frac{\kappa}{1-e^{-r \Delta}},
$$

and

$$
r V_{t}(j)=\left(\frac{1-\alpha}{\mu}\right)^{\frac{1}{\alpha}} e^{\frac{\pi}{\alpha} \Delta}\left(\mu e^{-\pi \Delta}-1\right) l_{y t},
$$

respectively. The final-goods market clearing condition is $c_{t}+z_{t}+x_{t}=y_{t}$, where

$$
\begin{gathered}
z_{t}=\kappa \sum_{s=0}^{\infty} g N_{t} e^{-g s \Delta}=\frac{g \mathcal{K}}{1-e^{-g \Delta}} N_{t}, \\
x_{t}=(1-\alpha)^{\frac{1}{\alpha}} l_{y} \frac{g N_{t}}{1-e^{-g \Delta}} \Phi(\Delta),
\end{gathered}
$$




$$
y_{t}=\frac{w_{t} l_{y t}}{\alpha}=(1-\alpha)^{\frac{1-\alpha}{\alpha}} \frac{g N_{t}}{1-e^{-g \Delta}} \Omega(\Delta) l_{y t},
$$

where $\Omega(\Delta)$ and $\Phi(\Delta)$ are denoted in the benchmark model. The model is reduced to a system of two equations and endogenous variables $\Delta$ and $g$. Combining (C.I) with (C.4), together with the expression of wage rate in (C.7), yields the first equation such that

$$
\frac{\alpha \mu g}{1-\alpha} \frac{e^{\left(\frac{1-\alpha}{\alpha} \pi-g\right) \Delta}-1}{\frac{1-\alpha}{\alpha} \pi-g}=\frac{1-e^{-g \Delta}}{\rho+g}(\beta-g) e^{\frac{\pi}{\alpha} \Delta}\left(\mu e^{-\pi \Delta}-1\right)
$$

Inserting (C.3) into (C.4) yields the second equation such that

$$
\frac{\mu\left(1-e^{-\left(\rho+g-\frac{1-\alpha}{\alpha} \pi\right) \Delta}\right)}{\rho+g-(1-\alpha) \pi / \alpha}-\frac{1-e^{-\left(\rho+g-\frac{\pi}{\alpha}\right) \Delta}}{\rho+g-\pi / \alpha}-\frac{\left(1-e^{-(\rho+g) \Delta}\right) e^{\frac{\pi \Delta}{\alpha}}\left(\mu e^{-\pi \Delta}-1\right)}{\rho+g}=\left(\frac{\mu}{1-\alpha}\right)^{\frac{1}{\alpha}} \frac{\kappa}{1-g / \beta},
$$

where the steady-state conditions $g=\dot{N}_{t} / N_{t}=\beta l_{r}$ and $l_{r}+l_{y}=1$ have been applied.

In this knowledge-based framework, inflation affects income inequality only in the way of changing the economic growth rate and thus the real interest rate (i.e., $r_{t}$ ), whereas leaving $a_{t} / w_{t}=V_{t} / w_{t}=1 / \beta$ unchanged. That is, the channel via the asset-value effect is silent. This feature is similar to that in Zheng (2020). Moreover, in this case, higher inflation can either stimulate or depress economic growth, which depends on two opposing forces. On the one hand, higher inflation induces firms to change prices more frequently and pay more menu costs, which in turn reduces the firm value and discourages innovation and growth. On the other hand, higher inflation decreases the demand of production labor and leads to a reallocation of labor to the $\mathrm{R} \& \mathrm{D}$ sector. As a result, the equilibrium (productivity-adjusted) wage rate (i.e., $w_{t} / N_{t}$ ) tends to rise and the free-entry condition implies an increase in the firm value, which encourages innovation and growth. Therefore, the effect of inflation on income inequality, which is completely determined by the growth effect of inflation, can be negative (positive) if the former force dominates (is dominated by) the latter force.

\section{References}

Arawatari, R., Hori, T. and Mino, K. (2018). On the nonlinear relationship between inflation and growth: A theoretical exposition. Journal of Monetary Economics, 94, 79- 93.

Chu, A. C. and CozzI, G. (2018). Effects of patents versus r\&d subsidies on income inequality. Review of Economic Dynamics, 29, 68-84.

- , - FAN, H., Furukawa, Y. and LiaO, C.-H. (2019). Innovation and inequality in a monetary schumpeterian model with heterogeneous households and firms. Review of Economic Dynamics, 34, 141 - 164. 
Coibion, O., Gorodnichenko, Y., KuenG, L. and Silvia, J. (2017). Innocent bystanders? monetary policy and inequality. Journal of Monetary Economics, 88, 70-89.

GoH, A.-T. and Olivier, J. (2002). Optimal patent protection in a two-sector economy. International Economic Review, 43 (4), 1191-1214.

Midrigan, V. (2011). Menu costs, multiproduct firms, and aggregate fluctuations. Econometrica, 79 (4), 1139-1180.

OiKawa, K. and UedA, K. (2018). The optimal inflation rate under schumpeterian growth. Journal of Monetary Economics, 100, 114-125.

Piketty, T., Saez, E. and Stantcheva, S. (2014). Optimal taxation of top labor incomes: A tale of three elasticities. American Economic Journal: Economic Policy, 6 (1), 230-271.

Rivera-Batiz, L. A. and Romer, P. M. (1991). International trade with endogenous technological change. European economic review, 35 (4), 971-1001.

Romer, P. M. (1990). Endogenous technological change. Journal of Political Economy, 98 (5, Part 2), S71-S102.

Saez, E. and Zucman, G. (2016). Wealth inequality in the united states since 1913: Evidence from capitalized income tax data. Quarterly Journal of Economics, 131 (2), 519-578.

SHESHINSKI, E. and WeIss, Y. (1977). Inflation and costs of price adjustment. Review of Economic Studies, 44 (2), 287-303.

ZHENG, Z. (2020). Inflation and income inequality in a schumpeterian economy with menu costs. Economics Letters, 186, 108524 . 